\title{
Supporting Information \\ Rate induced solubility and suppression of the first-order phase transition in olivine $\mathrm{LiFePO}_{4}$
}

Xiaoyu Zhang ${ }^{1}$, Martijn van Hulzen ${ }^{1}$, Deepak P. Singh ${ }^{1}$, Alex Brownrigg ${ }^{2}$, Jonathan P. Wright ${ }^{2}$, Niels H. van Dijk ${ }^{1}$ and Marnix Wagemaker ${ }^{1 *}$

${ }^{1}$ Department of Radiation Science and Technology, Delft University of Technology, Mekelweg 15, 2629JB, Delft, The Netherlands

${ }^{2}$ European Synchrotron Radiation Facility, 6 rue Jules Horowitz,

BP 220, 38043 Grenoble Cedex, France

*corresponding author e-mail: $\underline{\text { m.wagemaker@tudelft.nl }}$

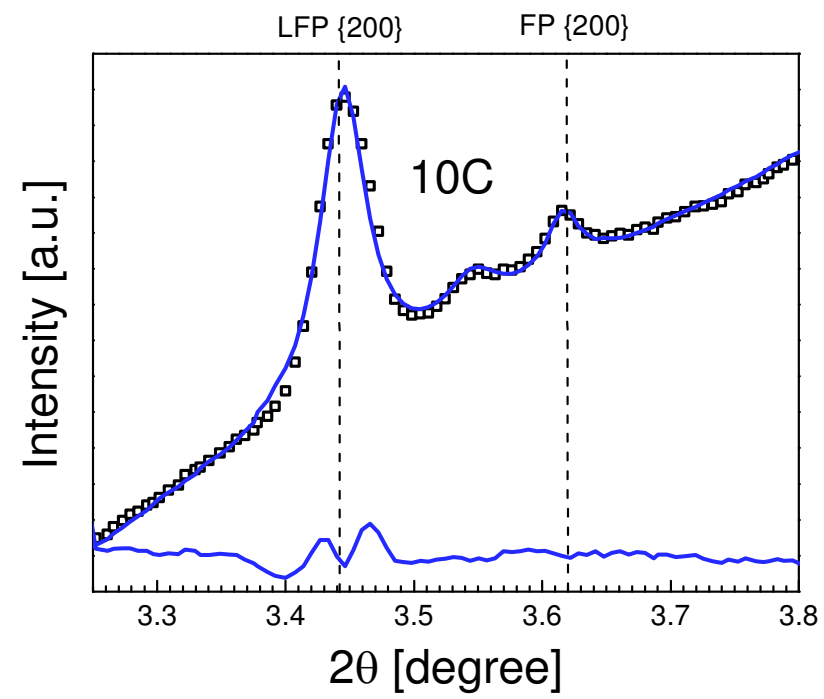

Figure S1. Diffraction pattern at approximately 5\% state of charge during $10 \mathrm{C}$ charging displaying a weak intermediate reflection with the a-lattice parameter equal to $10.03 \AA$ representing a meta stable composition between the FP and LFP phase. 

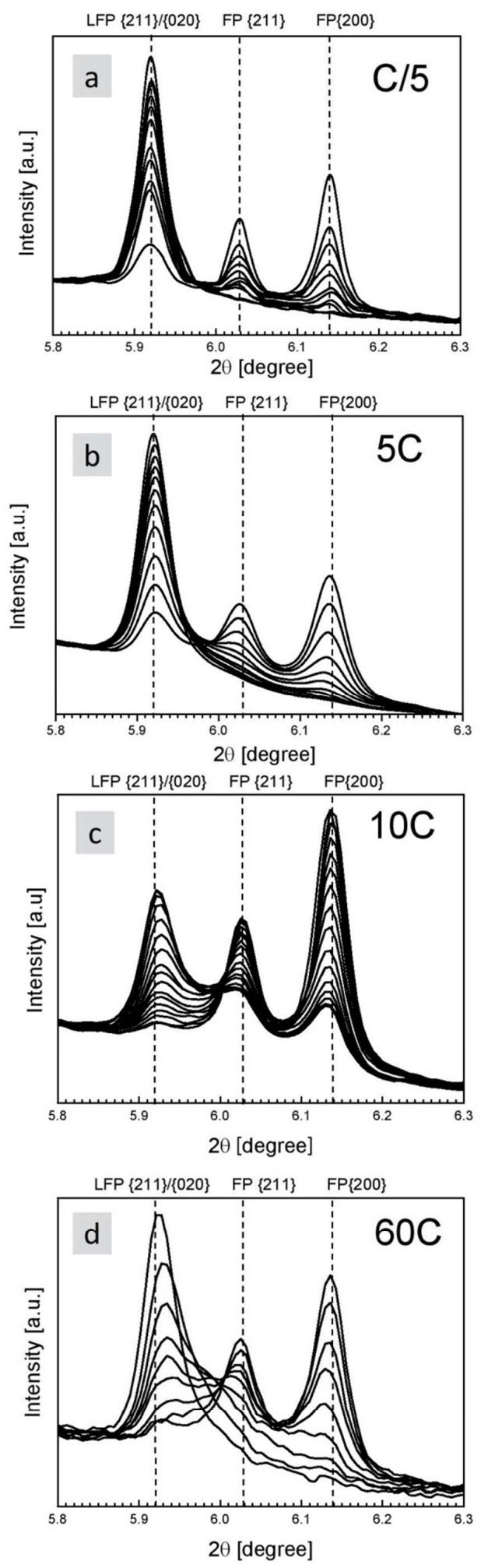

Figure S2. (a-d) Evolution of the $\{211\}$ and $\{020\}$ reflections during $C / 5,5 C, 10 C$ and $60 C$ charging. The vertical lines indicate the equilibrium $\{211\}$ and $\{020\}$ position reflecting the equilibrium unit cell parameters in both the Li-rich (LFP) and Li-poor (FP) phases. 


\section{Methods}

Sample preparation. The starting material was carbon coated $\mathrm{LiFePO}_{4}$ from Phostech with an average particle size of $140 \mathrm{~nm}$. Regular $\mathrm{LiFePO}_{4}$ cathodes were prepared through mixing a slurry of $\mathrm{LiFePO}_{4}$, Carbon Black (Super P), PVDF, (polyvinylidene fluoride, Solvay) in NMP (Nmethylpyrrolidone, with a mass ratio of the active material $\left(\mathrm{LiFePO}_{4}\right)$, carbon black (SuperP) and binder (PVDF) of 80:10:10. In addition carbonate template electrodes were prepared to reach high capacities at high rates as reported recently ${ }^{36}$. For the electrodes, $40 \mathrm{wt} \%$ $\mathrm{NaHCO}_{3}$ (Aldrich) is added to the electrode slurry mixing, a large part which is lost by dissolution in the solvent. The final slurry was casted on carbon coated Aluminium current collectors by doctor blading. The coatings were dried on a heater plate under air at approximately $155{ }^{\circ} \mathrm{C}$ overnight followed by drying under vacuum at around $60{ }^{\circ} \mathrm{C}$ for more than 24 hours. The resulting coatings were pressed using a roller hand press to enhance the electronic contact. After mechanical compaction the $\mathrm{NaHCO}_{3}$ templated electrodes were washed in demineralized water that reacts with the $\mathrm{NaHCO}_{3}$ to form $\mathrm{NaOH}$ and gaseous $\mathrm{CO}_{2}$, resulting in a an electrode where the interconnectivity of the porosity in the electrodes is improved as demonstrated previously ${ }^{36}$. Finally, the electrodes are dried for at least 3 hours under vacuum at $100{ }^{\circ} \mathrm{C}$. The results are reported on electrodes with loading density between 2 and $4 \mathrm{mg} / \mathrm{cm}^{2}$ and with a thickness of approximately 10-20 $\mu \mathrm{m}$.

Battery preparing and testing. The electrode were assembled in 'coffee-bag' type cells built following the plastic $\mathrm{Li}$-ion cell procedure. All the samples were assembled under argon atmosphere $\left(<0.1 \mathrm{ppm} \mathrm{O} \mathrm{O}_{2} / \mathrm{H}_{2} \mathrm{O}\right)$. The electrodes were separated by glass microfiber filters (Whatman) with a few droplets of $1 \mathrm{~mol} /$ liter LiPF6 (EC:DMC 1:1, Novolyte) electrolyte. All the electrochemical tests were performed galvanostatically within a voltage window of 4.3 and $2.5 \mathrm{~V}$ vs $\mathrm{Li} / \mathrm{Li}^{+}$using an Autolab PGSTAT302N potentiostat/galvanostat.

In situ synchrotron X-ray diffraction. The in situ XRD measurements were performed at the ID11 beam line of the ESRF (France), using a monochromatic X-ray beam with $\lambda=0.30996 \AA$ ( $E=40 \mathrm{keV}$ ), a beam size of $50 \mu \mathrm{m}$ and a FReLoN2k CCD detector. During exposure the sample was continuously rotated around the axis perpendicular to the $\mathrm{X}$-ray beam over an angular range of $1^{\circ}$ with an exposure time of $10 \mathrm{~s}(C / 5,5 C), 5 \mathrm{~s}(10 \mathrm{C})$ and $1 \mathrm{~s}(60 \mathrm{C})$. The sloping background in the diffraction patterns is due to small angle and incoherent scattering of the plastic of the pouch cells and the electrolyte and separator. 